EPJ Web of Conferences 113, 04026 (2016)

DOI: $10.1051 /$ epjconf/201611304026

C) Owned by the authors, published by EDP Sciences, 2016

\title{
Hadronic parity violation in few-body systems
}

\author{
M. R. Schindler ${ }^{1, a}$ \\ ${ }^{1}$ Department of Physics and Astronomy, University of South Carolina, Columbia, SC 29208, USA
}

\begin{abstract}
.
Efforts to describe hadronic parity violation in few-nucleon systems using effective field theories are described. Particular emphasis is given to observables in two- and threenucleon systems and their relation to ongoing experimental efforts. The role of parityviolating three-nucleon interactions is also discussed.
\end{abstract}

\section{Introduction}

Parity-violating (PV) interactions of hadrons are the manifestation of an interplay of strong and weak interactions between quarks inside the hadrons (for recent reviews see, e.g., Refs. [1, 2]). For the case of nucleon-nucleon (NN) interactions, the PV component is expected to be suppressed by a factor of about $10^{-6}$ to $10^{-7}$ compared to the dominant parity-conserving (PC) part. These small effects can be isolated by considering observables that would vanish if parity was conserved. Examples of such observables include longitudinal and angular asymmetries in scattering with polarized beams or targets. Parity-violating effects can be enhanced by up to several orders of magnitude in more complex nuclei with closely-spaced energy levels. However, the theoretical description and interpretation in terms of two- and three-nucleon interactions becomes difficult and can introduce uncontrolled errors. The development of high-intensity sources and advances in the control of systematics have led recent efforts to be focussed on the detection of parity violation in few-nucleon systems. For these systems, the relation to the underlying nucleon-nucleon interactions is much easier to establish. Most of the corresponding experiments are related to processes at very low energies, e.g., the capture of cold neutrons. At these energies, effective field theories (EFT) provide a model-independent framework to analyze and interpret data, and to give theoretical uncertainty estimates.

Parity-violating interactions between nucleons originate in weak quark-quark interactions inside nucleons. While the weak interactions at the quark level are well-understood, their manifestation at the hadronic level is complicated by nonperturbative QCD confining the quarks into hadrons. Because the weak interactions between quarks are very short-ranged, the PV nucleon forces are sensitive to short-distance quark-quark correlations inside the nucleon. Hadronic parity violation can therefore be used to gain a better understanding of nonperturbative strong interactions. While the EFT analysis to be discussed does not directly relate hadronic parity violation to standard model degrees of freedom and their interactions, any quark-level calculation of hadronic parity violation must be consistent with the EFT results. One possible approach is given by lattice QCD [3].

\footnotetext{
ae-mail: mschindl@mailbox.sc.edu
} 


\section{Parity violation in pionless EFT}

At energies well below the pion mass, it is possible to construct a theory in which the only dynamical degrees of freedom are nucleons, while pions and all heavier particles are "integrated out" and do not appear explicitly. In this so-called pionless EFT $(\mathrm{EFT}(\not{t}))$, the interactions between nucleons are described in terms of contact terms with an increasing number of derivatives and nucleon fields. Each contact term is accompanied by a so-called low-energy constant (LEC) that encodes the short-distance details that have been integrated out. Observables are calculated order-by-order in an expansion in a small parameter that is based on a ratio of scales. Pionless EFT has been very successfully applied in the PC sector, for reviews see, e.g., Refs. [4-6].

At leading order $(\mathrm{LO})$ in EFT( $\not$ ), parity violation can be described in terms of five operators that parameterize S- to P-wave transitions [7-9]. The corresponding Lagrangian can be written as [10]

$$
\begin{aligned}
\mathcal{L}_{P V}=- & {\left[g^{\left({ }^{3} S_{1}-{ }^{1} P_{1}\right)} d_{t}^{i \dagger}\left(N^{T} \sigma_{2} \tau_{2} i \stackrel{\leftrightarrow}{D}{ }_{i} N\right)+g_{(\Delta I=0)}^{\left(1 S_{0}-3 P_{0}\right)} d_{s}^{A \dagger}\left(N^{T} \sigma_{2} \vec{\sigma} \cdot \tau_{2} \tau_{A} i \stackrel{\leftrightarrow}{D} N\right)\right.} \\
& +g_{(\Delta I=1)}^{\left({ }^{1} S_{0}-{ }^{3} P_{0}\right)} \epsilon^{3 A B} d_{s}^{A \dagger}\left(N^{T} \sigma_{2} \vec{\sigma} \cdot \tau_{2} \tau^{B} \stackrel{\leftrightarrow}{D} N\right)+g_{(\Delta I=2)}^{\left({ }^{1} S_{0}{ }^{3} P_{0}\right)} \mathcal{I}^{A B} d_{s}^{A \dagger}\left(N^{T} \sigma_{2} \vec{\sigma} \cdot \tau_{2} \tau^{B} i \stackrel{\leftrightarrow}{D} N\right) \\
& \left.+g^{\left({ }^{3} S_{1}-{ }^{3} P_{1}\right)} \epsilon^{i j k} d_{t}^{i \dagger}\left(N^{T} \sigma_{2} \sigma^{k} \tau_{2} \tau_{3} \stackrel{\leftrightarrow}{D^{j}} N\right)\right]+ \text { h.c., }
\end{aligned}
$$

where $d_{t}$ and $d_{s}$ represent auxiliary fields for two-nucleon states in the ${ }^{3} S_{1}$ and ${ }^{1} S_{0}$ channels, respectively [11], $a O \stackrel{\leftrightarrow}{D}_{i} b=a O D_{i} b-\left(D_{i} a\right) O b$, with $O$ some spin-isospin-operator, and $\mathcal{I}=\operatorname{diag}(1,1,-2)$. The five LECs $g^{(X-Y)}$ encode the short-distance details of the interactions and cannot be determined within the EFT framework. In principle, they can be calculated in terms of standard-model parameters. However, this requires a nonperturbative QCD calculation, which has not been feasible so far. As an alternative, the LECs can be determined from data once a sufficient number of experiments and corresponding calculations has been performed.

\section{Two-nucleon systems}

Two-nucleon systems provide the theoretically simplest cases to study PV nucleon-nucleon interactions. The PV longitudinal asymmetry $A_{L}$ in scattering of a polarized beam on an unpolarized target is defined as

$$
A_{L}=\frac{\sigma_{+}-\sigma_{-}}{\sigma_{+}+\sigma_{-}}
$$

where $\sigma_{ \pm}$denotes the total cross section for scattering with beam helicity \pm 1 . At LO in $\operatorname{EFT}(\not t)$, the results for various beam and target nucleons, adjusted to the conventions of Eq. (1), are [9]

$$
\begin{aligned}
A_{L}^{n n}= & -\sqrt{\frac{32 M}{\pi}} p\left(g_{(\Delta I=0)}^{\left({ }^{1} S_{0}{ }^{3} P_{0}\right)}-g_{(\Delta I=1)}^{\left({ }^{1} S_{0}{ }^{3} P_{0}\right)}+g_{(\Delta I=2)}^{\left(1{ }^{1} S_{0}{ }^{3} P_{0}\right)}\right), \\
A_{L}^{p p}= & -\sqrt{\frac{32 M}{\pi}} p\left(g_{(\Delta I=0)}^{\left({ }^{1} S_{0}{ }^{3} P_{0}\right)}+g_{(\Delta I=1)}^{\left({ }^{1} S_{0}{ }^{3} P_{0}\right)}+g_{(\Delta I=2)}^{\left({ }^{1} S_{0}-^{3} P_{0}\right)}\right), \\
A_{L}^{n p}= & -\sqrt{\frac{32 M}{\pi}} p \frac{\frac{d \sigma^{1} S_{0}}{d \Omega}}{\frac{d \sigma^{1} S_{0}}{d \Omega}+3 \frac{d \sigma^{3} S_{1}}{d \Omega}}\left(g_{(\Delta I=0)}^{\left({ }^{1} S_{0}{ }^{3} P_{0}\right)}-2 g_{(\Delta I=2)}^{\left({ }^{1} S_{0}{ }^{3} P_{0}\right)}\right) \\
& -\sqrt{\frac{32 M}{\pi}} p \frac{\frac{d \sigma^{3} S_{1}}{d \Omega}}{\frac{d \sigma^{1} S_{0}}{d \Omega}+3 \frac{d \sigma^{3} S_{1}}{d \Omega}}\left(g^{\left({ }^{3} S_{1}-^{-1} P_{1}\right)}+2 g^{\left({ }^{3} S_{1}-{ }^{3} P_{1}\right)}\right),
\end{aligned}
$$




\section{$21^{\text {st }}$ International Conference on Few-Body Problems in Physics}

where $M$ denotes the nucleon mass and $p$ is the nucleon momentum in the center-of-mass frame. The result of Eq. (4) neglects Coulomb effects. However, as shown in Ref. [9], they only amount to a correction of about $3 \%$ for the lowest energy that has been experimentally considered.

Parity-violating interactions induce a rotation of the nucleon spin in the transmission of a perpendicularly polarized beam through an unpolarized target. In the case of a neutron beam and a hydrogen target, the rotation angle per unit length at NLO is given by [12]

$$
\frac{1}{\rho} \frac{d \phi_{\mathrm{PV}}^{n p}}{d l}=4 \sqrt{2 \pi M}\left(\frac{2 g^{\left({ }^{3} S_{1}-{ }^{3} P_{1}\right)}+g^{\left({ }^{3} S_{1}{ }^{1} P_{1}\right)}}{\gamma_{t}} \frac{Z_{t}+1}{2}+\frac{g_{(\Delta I=0)}^{\left({ }^{1} S_{0}{ }^{3} P_{0}\right)}-2 g_{(\Delta I=2)}^{\left({ }^{1} S_{0}{ }^{3} P_{0}\right)}}{\gamma_{s}} \frac{Z_{s}+1}{2}\right) .
$$

Here, $\rho$ denotes the target density, $\gamma_{t / s}$ are the poles in the NN scattering amplitude in the S-wave spintriplet/spin-singlet channel, and $Z=\left(1-\gamma_{t / s} r_{t / s}\right)^{-1}$ with $r_{t / s}$ the effective range in the corresponding channel.

Additional observables are available when interactions with photons are considered. In the radiative capture of polarized neutrons on protons $(\vec{n} p \rightarrow d \gamma)$, the direction of the outgoing photon can be used to define an angular asymmetry $A_{\gamma}$. Determination of $A_{\gamma}$ has been the goal of the NPDGamma collaboration at the Spallation Neutron Source (SNS) at Oak Ridge National Laboratory [13]. With data taking being completed, the result of this measurement is eagerly being awaited. In EFT( $\not$ ) at $\mathrm{LO}$, the result is given by $[10,14]$

$$
A_{\gamma}=\frac{4}{3} \sqrt{\frac{2}{\pi}} \frac{M^{\frac{3}{2}}}{\kappa_{1}\left(1-\gamma_{t} a_{s}\right)} g^{\left(3 S_{1}-{ }^{3} P_{1}\right)},
$$

with $a_{s}$ the scattering length in the ${ }^{1} S_{0}$ channel and $\kappa_{1}$ the isovector nucleon anomalous magnetic moment.

The capture of unpolarized neutrons provides an independent and complementary observable in the induced circular polarization $P_{\gamma}$ of the outgoing photons. For reversed kinematics, it is identical to the PV asymmetry $A_{L}^{\gamma}$ in the deuteron breakup reaction with polarized photons $(\vec{\gamma} d \rightarrow n p)$,

$$
A_{L}^{\gamma}=\frac{\sigma_{+}-\sigma_{-}}{\sigma_{+}+\sigma_{-}}
$$

where $\sigma_{ \pm}$stands for the total breakup cross section for photon helicities \pm 1 . A measurement of $A_{L}^{\gamma}$ would require a high-intensity photon source with good control of the beam. The possibility of performing this experiment at an upgraded HIGS facility at the Triangle Universities Nuclear Laboratory has been under consideration. The PV asymmetry was calculated to NLO in EFT( $\not$ ) in Ref. [15]. Because the PV LECs have not been reliably determined, their values were related to different model estimates (see Ref. [15] for details). The energy dependence of $A_{L}^{\gamma}$ using three different estimates for the LECs is shown in Fig. 1. The asymmetry is maximum at threshold, where the denominator in Eq. (8) vanishes. To determine at which energy to best perform the experiment, a very simplistic figure of merit of the form

$$
f=\left(A_{L}^{\gamma}\right)^{2} \times \sigma(\gamma d \rightarrow n p)
$$

was considered. As shown in Fig. 2, for the chosen values of the PV LECs, the figure of merit is maximized for photon energies in the range $2.259 \mathrm{MeV}<k<2.264 \mathrm{MeV}$. It is important to note, though, that because of the uncertainty in the estimation of the LECs, the shown results are only representations of the possible values of $A_{L}^{\gamma}$ and should only be considered as order-of-magnitude estimates. 


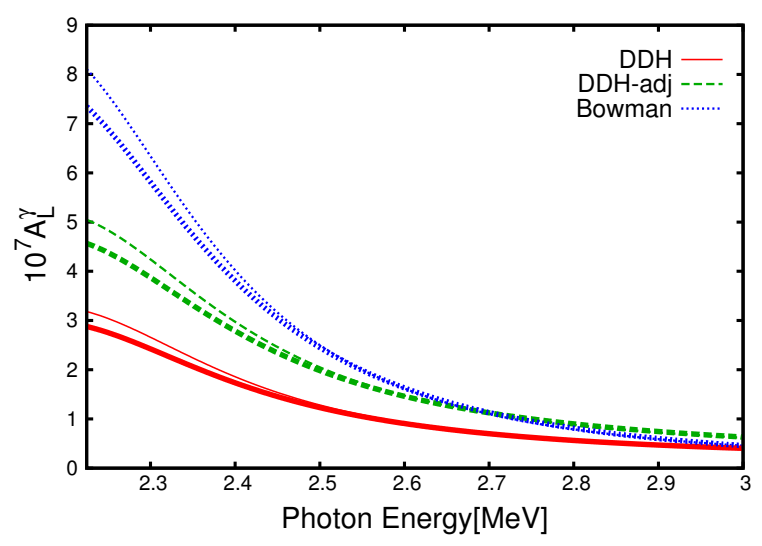

Figure 1. Energy dependence of the PV asymmetry $A_{L}^{\gamma}$ for various LEC estimates from Ref. [15]. The thin and thick lines correspond to different $\operatorname{EFT}(\not t)$ parametrizations and can be viewed as an indication of the theoretical uncertainty.

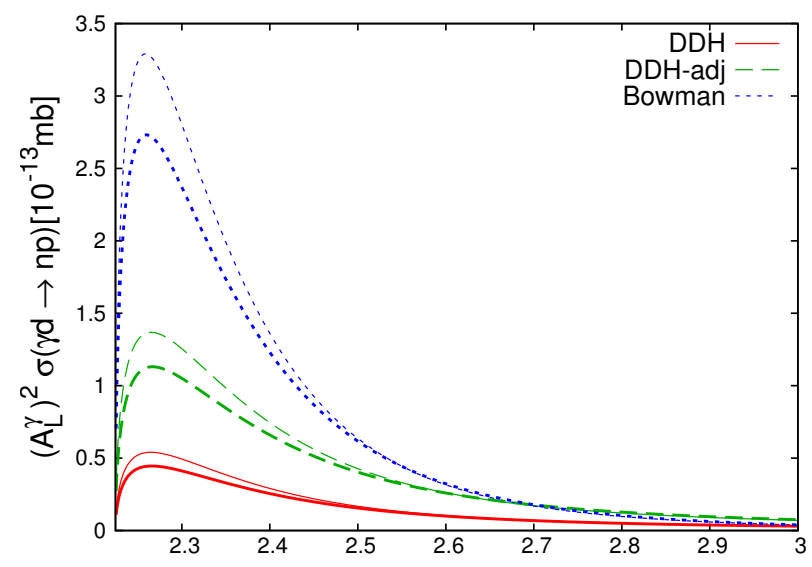

Figure 2. Figure of merit $f$ for various LEC estimates from Ref. [15]. The thin and thick lines correspond to different $\operatorname{EFT}(\not)$ parametrizations and can be viewed as an indication of the theoretical uncertainty.

\section{Three-nucleon systems}

In the PC sector of pionless EFT, a direct application of the power-counting rules does not predict three-nucleon forces at LO. However, as shown in Ref. [16], an unphysical cutoff dependence occurs in the scattering amplitude for spin-doublet neutron-deuteron scattering. This cutoff dependence can be removed by introducing a three-nucleon contact term already at LO. The corresponding LEC can be determined from the experimental value of, e.g., the three-nucleon binding energy or the neutrondeuteron scattering length. A similar possible enhancement of a PV three-nucleon interaction to LO would require an additional experimental result to determine the corresponding LEC. Given the scarcity of data in the few-nucleon sector, this would complicate the analysis of hadronic parity violation considerably. However, the analysis of PV nucleon-deuteron scattering of Ref. [17] showed that such an enhancement of a PV three-nucleon interaction does not occur at LO or NLO. Therefore, 
up to an accuracy of roughly $10 \%$, it is sufficient to analyze observables using only PV two-nucleon interactions.

The spin rotation angle for the transmission of a polarized neutron through a deuteron target was calculated in $\operatorname{EFT}(\not t)$ to NLO in Ref. [12]. The result is

$$
\begin{aligned}
& \frac{1}{\rho} \frac{d \phi_{\mathrm{PV}}^{n d}}{d l}=( {[16 \pm 1.6] g^{\left({ }^{3} S_{1}-{ }^{1} P_{1}\right)}+[34 \pm 3.4] g^{\left({ }^{3} S_{1}{ }^{3} P_{1}\right)} } \\
&\left.+[4.6 \pm 1.0]\left(3 g_{(\Delta I=0)}^{\left({ }^{1} S_{0}-3 P_{0}\right)}-2 g_{(\Delta I=1)}^{\left({ }^{1} S_{0}-3 P_{0}\right)}\right)\right) \frac{\mathrm{rad}}{\mathrm{MeV}^{\frac{1}{2}}}
\end{aligned}
$$

The theoretical uncertainties correspond to the most conservative of three error estimates based on power counting arguments, cutoff dependence, and different inputs used to fix the PC three-nucleon interaction.

\section{Few-nucleon systems}

A measurement of a PV asymmetry in the charge-exchange reaction $\vec{n}^{3} \mathrm{He} \rightarrow p^{3} \mathrm{H}$ is currently underway at the SNS. The corresponding calculation using an EFT with explicit pion degrees of freedom was performed in Ref. [18]. Experimental results also exist for the PV asymmetry in $\vec{p}^{4} \mathrm{He}$ scattering [19] and the neutron spin rotation angle in a ${ }^{4} \mathrm{He}$ target [20]. No calculations in terms of twoand three-nucleon interactions have so far been performed, but will form an important component in improving our understanding of hadronic parity violation.

\section{Conclusions and outlook}

Because of their origin in an interplay between short-distance weak interactions and nonperturbative QCD, PV interactions between nucleons provide a unique probe of our understanding of the standard model. Considering PV observables in few-nucleon systems avoids possible complications from nuclear structure. At very low energies, EFT( $\not$ ) provides a model-independent framework for the analysis and interpretation of hadronic parity violation. At LO, the PV nucleon-nucleon interactions can be described by five independent operators. The corresponding LECs encode the short-distance details of the underlying interactions. Once the LECs have been determined from comparison with experimental results, their values provide a nontrivial target that any calculation in the nonperturbative regime has to reproduce. A preliminary calculation of a PV pion-nucleon coupling using lattice QCD was performed in Ref. [3], and further work in this direction will be of great importance.

\section{Acknowledgements}

This work was supported by the US Department of Energy under Grant No. DE-SC0010300. I am grateful to H. W. Grießhammer, D. R. Phillips, R. P. Springer, and J. Vanasse for their collaboration and many stimulating discussions.

\section{References}

[1] W.C. Haxton, B.R. Holstein, Prog.Part.Nucl.Phys. 71, 185 (2013), 1303.4132

[2] M.R. Schindler, R.P. Springer, Prog.Part.Nucl.Phys. 72, 1 (2013), 1305.4190

[3] J. Wasem, Phys.Rev. C85, 022501 (2012), 1108.1151 
[4] S.R. Beane, P.F. Bedaque, W.C. Haxton, D.R. Phillips, M.J. Savage, In *Shifman, M. (ed.): At the frontier of particle physics, vol. $1^{*}$ pp. 133-269. (2000), nucl-th/0008064

[5] P.F. Bedaque, U. van Kolck, Ann. Rev. Nucl. Part. Sci. 52, 339 (2002), nucl-th/0203055

[6] L. Platter, Few Body Syst. 46, 139 (2009), 0904 . 2227

[7] S.L. Zhu, C.M. Maekawa, B.R. Holstein, M.J. Ramsey-Musolf, U. van Kolck, Nucl.Phys. A748, 435 (2005), nucl-th/0407087

[8] L. Girlanda, Phys. Rev. C77, 067001 (2008), 0804.0772

[9] D.R. Phillips, M.R. Schindler, R.P. Springer, Nucl. Phys. A822, 1 (2009), 0812 . 2073

[10] M.R. Schindler, R.P. Springer, Nucl. Phys. A846, 51 (2010), 0907. 5358

[11] D.B. Kaplan, Nucl. Phys. B494, 471 (1997), nucl-th/9610052

[12] H.W. Grießhammer, M.R. Schindler, R.P. Springer, Eur.Phys.J. A48, 7 (2012), 1109. 5667

[13] M.T. Gericke, R. Alarcon, S. Balascuta, L. Barron-Palos, C. Blessinger et al., Phys.Rev. C83, 015505 (2011)

[14] M.J. Savage, Nucl. Phys. A695, 365 (2001), nucl-th/0012043

[15] J. Vanasse, M.R. Schindler, Phys. Rev. C90, 044001 (2014), 1404.0658

[16] P.F. Bedaque, H.W. Hammer, U. van Kolck, Nucl. Phys. A676, 357 (2000), nucl-th/9906032

[17] H.W. Grießhammer, M.R. Schindler, Eur. Phys. J. A46, 73 (2010), 1007.0734

[18] M. Viviani, A. Baroni, L. Girlanda, A. Kievsky, L. Marcucci et al. (2014), 1403. 2267

[19] J. Lang, T. Maier, R. Muller, F. Nessi-Tedaldi, T. Roser et al., Phys.Rev.Lett. 54, 170 (1985)

[20] W.M. Snow, C.D. Bass, T.D. Bass, B.E. Crawford, K. Gan et al., Phys.Rev. C83, 022501 (2011) 